\title{
Nephrogenic Systemic Fibrosis: More Questions and Some Answers
}

\author{
S.K. Morcos ${ }^{a}$ H.S. Thomsen ${ }^{b}$ \\ a Department of Diagnostic Imaging, Northern General Hospital, Sheffield Teaching Hospitals NHS Foundation \\ Trust, Sheffield, UK; ${ }^{b}$ Department of Diagnostic Radiology, Copenhagen University Hospital, Herlev, and \\ Department of Diagnostic Sciences, Faculty of Health Sciences, University of Copenhagen, Copenhagen, Denmark
}

\section{Key Words}

Nephrogenic systemic fibrosis • Gadolinium-based contrast agents $\cdot$ Magnetic resonance imaging

\begin{abstract}
Nephrogenic systemic fibrosis (NSF) is a fibrosing disorder that may develop in patients who have advanced reduction in renal function. A causal relation between gadolinium $\left(\mathrm{Gd}^{3+}\right)$-based contrast agents (Gd-CA) and NSF is probable and is supported by the accumulating data in the literature. From those data, the prevalence of NSF is seen to be significantly higher after exposure to gadodiamide than any other gadolinium-based agent. Gd-CA are either linear or macrocyclic chelates and are available as ionic or non-ionic preparations. The molecular structure, whether cyclic or linear, and the ionicity determine the stability of Gd-CA. Linear chelates are flexible open chains which do not offer a strong binding to $\mathrm{Gd}^{3+}$. In contrast, the macrocyclic chelates offer a strong binding to $\mathrm{Gd}^{3+}$ by the virtue of being pre-organised rigid rings of almost optimal size to cage the $\mathrm{Gd}^{3+}$ atom. Non-ionic preparations are also less stable in comparison to the ionic ones, as the binding between $\mathrm{Gd}^{3+}$ and the negatively charged carboxyl groups is stronger than that with amides or alcohol in the non-ionic preparations. According to stability constants and kinetic measurements, the most stable Gd-CA is the ionic-macrocyclic chelate Gd-DOTA and the least stable agents are the non-ionic linear chelates ga-
\end{abstract}

\section{KARGER}

Fax +41613061234

E-Mail karger@karger.ch

www.karger.com

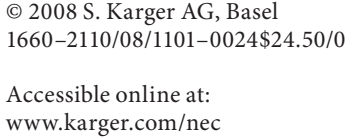

dodiamide and gadoversetamide. The stability of Gd-CA seems to be an important factor in the pathogenesis of NSF. Gd-CA of low stability are likely to undergo transmetallation and release free Gd ions that may deposit in tissues and attract circulating fibrocytes to initiate the process of fibrosis. There have been no cases of NSF reported in the peerreviewed literature after the exclusive use of the stable macrocyclic Gd-CA. This minireview covers the clinical and pathological features of NSF and updates the current understanding of the pathophysiology of this condition.

Copyright $\odot 2008$ S. Karger AG, Basel

\section{Introduction}

Nephrogenic systemic fibrosis (NSF) is a debilitating disorder that is seen in patients with a marked reduction in renal function. The world's first NSF case was identified in 1997 in California, USA, but the condition was first reported in the literature in 2000 by Cowper et al. [1]. Initially, the disease was given the name nephrogenic fibrosing dermopathy, but later, when reports demonstrated that the disease affects multiple organs and several tissues, the original name was replaced by the term 'nephrogenic systemic fibrosis' [2-4].

In January 2006, a report from Austria suggested a link between NSF and the administration of gadolinium $\left(\mathrm{Gd}^{3+}\right)$-based contrast agents (Gd-CA) [5]. A few months

Prof. S.K. Morcos

X-Ray Department

Northern General Hospital

Sheffield S5 7AU (UK)

Tel. +44 114271 4339, Fax +44 114261 1791, E-Mail sameh.morcos@sth.nhs.uk 
later, a report from Denmark documented a series of 13 patients who developed NSF following contrast-enhanced MRI examinations with Gd-CA [6]. Since then, a large number of reports have appeared in the literature linking Gd-CA with NSF [7].

This minireview is designed to offer a concise but comprehensive overview of NSF by answering - according to our current state of knowledge - important questions on this devastating disease.

\section{What Is NSF?}

NSF affects mainly patients with end-stage renal disease, including those on dialysis. It is characterised by the development of discoloured skin plaques that can be itchy and painful (fig. 1). The extremities and the trunk are affected but the head and neck are spared, with the exception of bilateral yellowed scleral plaques. Contractures of joints and complete loss of range of motion may occur. The severity of the disease varies from one patient to another and the fibrotic changes can be widespread, possibly affecting other organs such as the liver, lungs, muscles and heart $[4,8,9]$. In some cases, NSF causes serious physical disability, which can lead to patients requiring a wheelchair. Mortality in patients with multisystem involvement has also been reported $[4,9]$. The differential diagnosis of NSF is presented in table 1 [4].

\section{Pathological Findings}

Pathological changes seen on routine light microscopy of biopsies of affected skin vary with disease severity, ranging from subtle changes to marked thickening of the dermis. Thick collagen bundles, numerous CD34positive spindle cells (fibrocytes) and histiocytes are the prominent histological findings of NSF. Elastic fibres, mucin and elevated tissue levels of transforming growth factor- $\beta$ can also be detected. Calcification in vascular walls is a common feature. The fibrotic process may extend through the fascia into the underlying skeletal muscle, which becomes atrophic (fig. 1) [3, 4]. Deposits of Gd in the skin are not detected by routine histology stains.

\section{Is There a Causal Relation between NSF and Gd-CA?}

NSF was not identified before 1997, which suggests that it is a new disease that is probably due to exposure of patients with advanced chronic kidney disease (CKD) to

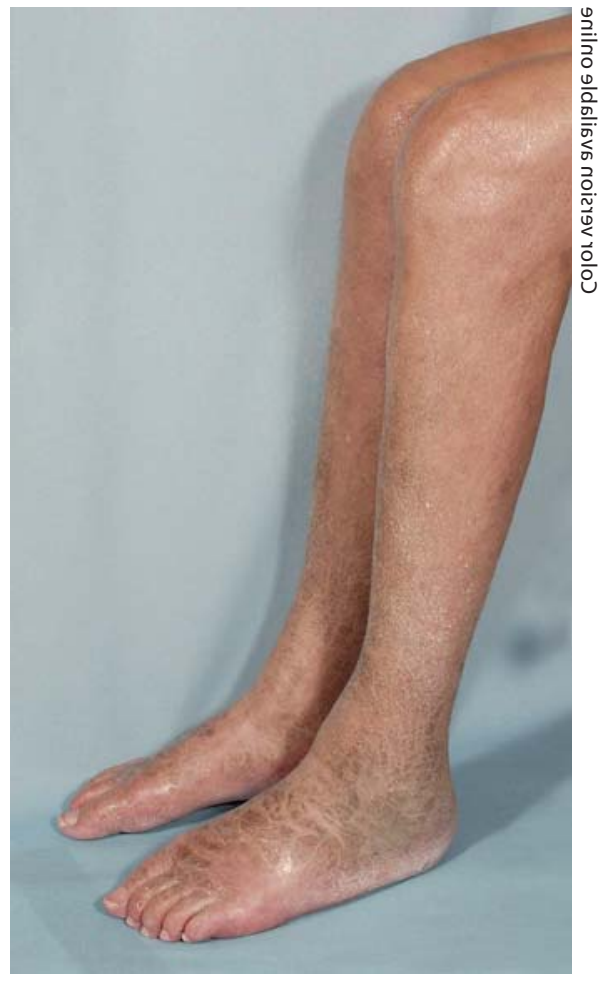

Fig. 1. Discoloured skin plaques affecting the feet and legs of a patient suffering from NSF. Atrophy of the calf muscles is also noticeable.

Table 1. Differential diagnosis of NSF [4]

\begin{tabular}{ll}
\hline Disease & $\begin{array}{l}\text { Characteristic features that differentiate } \\
\text { the disease from NSF }\end{array}$ \\
\hline $\begin{array}{l}\text { Lipodermato- } \\
\text { sclerosis }\end{array}$ & $\begin{array}{l}\text { Develops in patients who have oedema, } \\
\text { due to venous insufficiency } \\
\text { Involvement above the knee is rare }\end{array}$ \\
\hline Scleroderma & $\begin{array}{l}\text { Auto-antibodies (SCL-70 and anti- } \\
\text { nuclear antibodies) } \\
\text { Skin changes are seen on the forehead } \\
\text { Raynaud disease is a common feature }\end{array}$ \\
\hline Scleromyxoedema & $\begin{array}{l}\text { The face is commonly involved } \\
\text { Frequently associated with multiple } \\
\text { myeloma }\end{array}$ \\
\hline $\begin{array}{l}\text { Eosinophilic } \\
\text { fasciitis }\end{array}$ & $\begin{array}{l}\text { Peripheral eosinophilia is present } \\
\text { Underlying polyclonal hypergamma } \\
\text { globulinaemia is common }\end{array}$ \\
\hline $\begin{array}{l}\text { Chronic graft- } \\
\text { versus-host disease }\end{array}$ & $\begin{array}{l}\text { Similar to NSF, but the clinical setting is } \\
\text { characteristic (e.g. after allogeneic stem } \\
\text { cell transplantation) }\end{array}$ \\
\hline
\end{tabular}

Nephron Clin Pract 2008;110:c24-c32 
a new medication, infectious agent or toxin. It is of interest that over the last decade patients with CKD were frequently referred for contrast-enhanced magnetic resonance examinations, including magnetic resonance angiography, without undue concern. Clinical data up to 2006 demonstrated no serious adverse effects of Gd-CA in this group of patients and the reports on the general safety of these agents were extremely reassuring. Since January 2006, numerous published case series in the medical literature have confirmed a very strong association of NSF with exposure to Gd-CA. Furthermore, several reports published prior to 2006 have been updated to indicate their association with Gd-CA $[7,10]$. As of February 1,2008 , there have been 190 biopsy-proven cases of NSF associated with Gd-CA published in the peer-reviewed literature [7]. The onset of the disease varies from a few days to a few months after exposure to Gd-CA; a median time of 14 days (range 1-53 days) from exposure was reported in one study [8]. In some patients a period of a year or more between Gd-CA exposure and development of NSF has been claimed. Severe cases are usually associated with the large doses used for magnetic resonance angiography or after multiple exposures, but the disease has also been reported after a standard dose of Gd-CA [8].

\section{Can All Gd-CA Cause NSF?}

The vast majority of NSF cases have been associated with gadodiamide (Omniscan, GE Healthcare, USA). Smaller percentages of cases have been directly linked to gadopentetate dimeglumine (Magnevist, Bayer Schering, Germany), gadoversetamide (Optimark, Covidien, USA). Only 5 cases of NSF have been published that have no reliable Gd-CA exposure [7]. However, the skin biopsies of these 5 patients have not been examined for the presence of gadolinium. A summary of cases of NSF reported in the literature is presented in table 2 [7]. The frequent association with gadodiamide was also observed in cases reported to drug safety regulatory authorities in the USA and Europe, as well as in the records of the International Center for Nephrogenic Fibrosing Dermopathy Research at Yale University, USA. The frequent association of NSF with Omniscan (83\% of cases reported in the literature) cannot be explained by market share, which was around $26 \%$ in the USA from July 2005 to September 2007. Magnevist had the highest market share in the USA, which was about $54 \%$ in the same period [11].

The epidemiology of NSF associated with Gd-CA suggests that the stability of the Gd chelates is likely to be an important factor in the pathogenesis of this condition
Table 2. Biopsy-proven cases of NSF associated with Gd-CA published in the peer-reviewed literature up to February 2008 [7]

\begin{tabular}{lc}
\hline Gd-CA & $\begin{array}{l}\text { Number } \\
\text { of patients }\end{array}$ \\
\hline Omniscan & 157 \\
Magnevist & 8 \\
Optimark & 3 \\
Unspecified Gd-CA & 18 \\
Confounded cases with $>$ 1 Gd-CA & 4 \\
\hline
\end{tabular}

No cases of NSF in the peer-reviewed medical literature have been reported to be associated with macrocyclic agents. There have been 23 cases (20 Magnevist, 3 Omniscan) of NSF but without skin biopsy reported in the literature.

$[12,13]$. Molecules of low stability, such as gadodiamide, are prone to spontaneous dissociation and transmetallation with endogenous ions, leading to the release of free $\mathrm{Gd}$ ions $\left(\mathrm{Gd}^{3+}\right)$ which may deposit in tissues and initiate the process of fibrosis [12].

\section{What Do Stability of Gd-CA and Transmetallation}

Mean?

Extracellular Gd-CA are all chelates containing $\mathrm{Gd}^{3+}$. Stability refers to the relative tendency of the $\mathrm{Gd}^{3+}$ to remain coordinated (attached) to the chelating agent. Free $\mathrm{Gd}^{3+}$ is highly toxic and can cause splenic degeneration, central lobular necrosis of the liver, enzyme inhibition, calcium channel blocking and a variety of haematological abnormalities [14]. Therefore, to avoid its toxic effects, it is crucially important that $\mathrm{Gd}^{3+}$ should be strongly attached to a chelate. There are 7 extracellular Gd-CA currently available for clinical use (table 3) [12-14]. The configuration of the Gd-CA molecule is either linear or cyclic and they are available as ionic or non-ionic preparations. There are differences in the chemical stability of these agents and their liability to release free gadolinium ions. The binding to $\mathrm{Gd}^{3+}$ is weak in the non-ionic linear molecules, whereas the cyclic molecules offer better protection and binding to $\mathrm{Gd}^{3+}$ [12-14].

The following measurements are used to assess the stability of the chelate molecules: the thermodynamic stability constant at $\mathrm{pH} 11$, conditional stability at $\mathrm{pH} 7.4$ and kinetic stability [dissociation half-life under very acidic conditions ( $\mathrm{pH}$ 1)]. The higher the value of these measurements, the higher is the stability of the molecule. In addition, the presence of a large amount of excess chelate in the Gd-CA preparation is an indirect marker of the 
Table 3. Physicochemical characteristics of clinically available extracellular Gd-CA [12-14]

\begin{tabular}{|c|c|c|c|c|c|}
\hline Extracellular Gd-CA & Type & $\begin{array}{l}\text { Thermodynamic } \\
\text { stability constant }\end{array}$ & $\begin{array}{l}\text { Conditional } \\
\text { stability }\end{array}$ & $\begin{array}{l}\text { Amount of excess } \\
\text { chelate, } \mathrm{mg} / \mathrm{ml}\end{array}$ & $\begin{array}{l}\text { Kinetic stability (disso- } \\
\text { ciation half-life at pH 1.0) }\end{array}$ \\
\hline $\begin{array}{l}\text { Gadoversetamide, Gd-DTPA-BMEA } \\
\text { (Optimark, Covidien, USA) }\end{array}$ & non-ionic linear & 16.6 & 15 & 28.4 & not available \\
\hline $\begin{array}{l}\text { Gadodiamide, Gd-DTPA-BMA } \\
\text { (Omniscan, GE Healthcare, USA) }\end{array}$ & non-ionic linear & 16.9 & 14.9 & 12 & $35 \mathrm{~s}$ \\
\hline $\begin{array}{l}\text { Gadobutrol, Gd-BT-DO3A } \\
\text { (Gadovist, Bayer Schering Pharma AG, Germany) }\end{array}$ & non-ionic cyclic & 21.8 & not available & not available & $24 \mathrm{~h}$ \\
\hline $\begin{array}{l}\text { Gadoteridol, Gd-HP-DO3A } \\
\text { (Prohance, Bracco, Italy) }\end{array}$ & non-ionic cyclic & 23.8 & 17.1 & 0.23 & $3 \mathrm{~h}$ \\
\hline $\begin{array}{l}\text { Gadopentetate, Gd-DTPA } \\
\text { (Magnavist, Bayer Schering Pharma AG, Germany) }\end{array}$ & ionic linear & 22.1 & 18.1 & 0.4 & $10 \mathrm{~min}$ \\
\hline $\begin{array}{l}\text { Gadobenate, Gd-BOPTA } \\
\text { (Multihance, Bracco, Italy) }\end{array}$ & ionic linear & 22.6 & 18.4 & none & not available \\
\hline $\begin{array}{l}\text { Gadoterate, Gd-DOTA } \\
\text { (Dotarem, Guerbet, France) }\end{array}$ & ionic cyclic & 25.8 & 18.8 & none & $>1$ month \\
\hline
\end{tabular}

Table 4. Dissociation half-life of Gd-CA under controlled laboratory conditions [15]

\begin{tabular}{llll}
\hline & $\mathrm{pH} 1.2,37^{\circ} \mathrm{C}$ & $\mathrm{pH} 1,37^{\circ} \mathrm{C}$ & $\mathrm{pH} 1,25^{\circ} \mathrm{C}$ \\
\hline Dotarem & $85 \mathrm{~h}$ & $23 \mathrm{~h}$ & $338 \mathrm{~h}$ \\
Gadovist & $18 \mathrm{~h}$ & $7 \mathrm{~h}$ & $43 \mathrm{~h}$ \\
Prohance & $4 \mathrm{~h}$ & $1.6 \mathrm{~h}$ & $3.9 \mathrm{~h}$ \\
All linear chelates & $\mathrm{ND}$ & $\mathrm{ND}$ & $<5 \mathrm{~s}$ \\
\hline
\end{tabular}

ND $=$ Not determined

low stability of the molecule [12-15]. Considering the different stability measurements, the most stable molecule is the ionic cyclic chelate, which has the highest stability values and the longest dissociation half-life (table 3). In contrast, the least stable preparations are the non-ionic linear chelates which have the lowest thermodynamic and kinetic stability values and the highest amount of excess chelate in comparison to the other types of Gd-CA (table 3) [12-14]. A recent study evaluated dissociation half-life of Gd-CA under controlled laboratory conditions and confirmed that the ionic macrocyclic chelate has the highest kinetic stability, followed by the non-ionic macrocyclic chelates. The linear chelates had the lowest kinetic stability in the study (table 4) [15]. The clinical importance of the differences in the kinetic stability measured in vitro between the ionic and non-ionic macrocy-

Nephrogenic Systemic Fibrosis clic agents remains to be established. So far, in vivo studies measuring gadolinium retention in tissues after $\mathrm{Gd}$ CA exposure as a marker of the stability of the contrast agent have not shown significant differences between the macrocyclic agents [14].

Transmetallation refers to the replacement of the metal ion in the chelate by another ion. In vivo, endogenous ions, particularly zinc which is readily available, can replace $\mathrm{Gd}^{3+}$ to form chelates which are excreted in urine. The free $\mathrm{Gd}^{3+}$ cations become attached to endogenous anions, such as phosphate, hydroxide, carbonate or citrate, to form insoluble salts which deposit in tissues [12-14]. Transmetallation of Gd-CA by zinc ions was shown in healthy volunteers by measuring zinc excretion in urine after intravenous administration of GdCA [16]. Gadodiamide caused the highest increase in zinc in the urine, followed by Magnevist. The macrocyclic agent Dotarem (Guerbet, France) caused minimal zinc excretion [16]. In vitro studies also demonstrated that, in contrast to the linear chelates, all macrocyclic chelates are insensitive to transmetallation by zinc ions [17].

\section{What Is the Pathophysiology of NSF?}

In patients with advanced CKD, the elimination halflife of Gd-CA can be prolonged to $30 \mathrm{~h}$ or more [18]. Patients on haemodialysis would require 3 consecutive di- 
alysis sessions over 6 days to remove $97 \%$ of the administered dose of Gd-CA from the body. Continuous ambulatory peritoneal dialysis for 20 days eliminates only $69 \%$ of the injected dose of Gd-CA [18]. Transmetallation and spontaneous dissociation of Gd-CA, leading to the release of free $\mathrm{Gd}^{3+}$ cations, are likely to occur when the Gd chelate remains in the body for a long period, as is the case in patients with end-stage renal disease, including those on dialysis [12]. It is reasonable to hypothesise that free $\mathrm{Gd}^{3+}$ cations become attached to endogenous anions, particularly phosphate, forming insoluble salts that deposit in tissues. These insoluble molecules are engulfed by local macrophages, which in turn release a range of cytokines, including transforming growth factor- $\beta_{1}$, that attract circulating fibrocytes and initiate the process of fibrosis $[12,19]$. There is evidence that tissue fibrosis in NSF is caused by circulating fibrocytes recruited from the circulation, rather than by proliferation of resident dendritic cells. In addition, hybridisation studies have showed a marked increase in transforming growth factor- $\beta_{1}$ mRNA levels in the skin and fascia of patients with NSF $[3,4]$.

What Are the Co-Factors Which May Contribute to the Pathogenesis of NSF?

Most patients with advanced CKD who are exposed to Gd-CA do not develop NSF, suggesting that other factors, such as inflammatory events, metabolic acidosis, epoetin dosing or high phosphate levels, may play a role. However, it remains unclear whether the variables presented below are independently associated with an increased risk of NSF or it is simply that the majority of them are associated with an increased likelihood of Gd-CA administration [20].

Inflammatory Events. Some reports suggested a possible association between recent surgical interventions, infection or thrombotic events and NSF [21]. However, this association is not consistent and was not observed in other reports [8].

Metabolic Acidosis. Although transmetallation and release of $\mathrm{Gd}^{3+}$ ions are more likely to occur in an acidic milieu, the association between acidosis and NSF has not been confirmed in several studies [8].

Erythropoietin Therapy. Initiation of recombinant human erythropoietin (EPO) therapy or an increase in dose may be associated with NSF, but the true nature of the relationship between EPO and NSF remains incompletely understood. EPO has been implicated because it may have fibrogenic potential: it stimulates the bone marrow, and a large number of bone-marrow-derived CD34 fibro- blasts infiltrate the dermis in NSF [22]. An association between NSF and EPO has not been consistently observed and a cause-and-effect relationship cannot be proven [20].

Hyperphosphataemia. Patients with end-stage renal disease are likely to have high levels of serum phosphate which would compete to separate the $\mathrm{Gd}^{3+}$ ion from its chelate, forming gadolinium phosphate that may deposit in tissues and initiate the fibrotic process. Gd deposition in NSF has been found to be restricted to areas with concomitant calcium phosphate deposition [23]. Marckmann et al. [8] also found that among CKD patients exposed to Gd-CA, those who developed NSF had higher serum calcium and phosphate levels than those who did not develop NSF. Further studies are required to elucidate the role of hyperphosphataemia in the pathogenesis of NSF.

Liver Transplantation. NSF has been described in liver transplant recipients in combination with advanced renal dysfunction. The patients required dialysis before or after transplantation, although some patients were no longer dialysis dependent at the onset of NSF [24, 25].

Absence of Treatment with Angiotensin-Converting Enzyme Inhibitors. Angiotensin-Converting Enzyme inhibitors have anti-fibrogenic properties and their absence may promote the development of NSF [26]. However, this has not been a consistent finding in patients with NSF [8].

\section{What Are the Experimental Data on the Role of Gd-CA in the Pathogenesis of NSF?}

\section{In vivo Studies}

A rat model of NSF associated with repeated (20 days) injection of a high dose $(2.5 \mathrm{mmol} / \mathrm{kg})$ of Gd-CA was reported by Sieber et al. in 2008 [27]. Skin lesions consistent with human NSF were observed as early as 8 days after starting non-formulated Gd-DTPA-BMA treatment and 20 days after starting formulated Gd-DTPA-BMA (Omniscan) solution, but not Gd-DTPA (Magnevist). The highest $\mathrm{Gd}^{3+}$ concentrations in the skin and the most advanced skin lesions were found in animals that received low-stability Gd-CA. These data are strongly supportive of a link between released $\mathrm{Gd}^{3+}$ due to low stability of chelates and the development of NSF-like skin lesions. It is of interest that in this study the rats had normal renal function, and in spite of the rapid elimination of the injected Gd-CA (biological half-life of Gd-CA in rats with normal renal function is approx. $20 \mathrm{~min}$ ) NSF-like skin 
lesions developed, suggesting that some dissociation of the Gd-CA and release of free $\mathrm{Gd}^{3+}$ occurs even in the presence of normal renal function. It is reasonable to conclude from the findings of Sieber et al. [27, 28] that multiple repeated injections of low-stability Gd-CA in the absence of renal impairment can lead to gradual accumulation of $\mathrm{Gd}^{3+}$ in tissues until it reaches a threshold level that triggers the fibrotic process. A previous study in humans has shown that $\mathrm{Gd}^{3+}$ deposition in bone occurs in patients with normal renal function. In this study, the $\mathrm{Gd}^{3+}$ retention in bone with gadodiamide (Omniscan) was 2-4 times more than gadoteridol (Prohance, Bracco, Italy; a non-ionic macrocyclic Gd-CA) [29]. Thus, from the previously quoted studies it is tempting to postulate that multiple administrations of large doses of low-stability Gd-CA may cause NSF even in patients with normal renal function, although this is unlikely in clinical practice.

Additional studies are required, preferably using animal models of CKD such as the 5/6th subtotal nephrectomy model, which would reproduce an in vivo situation comparable to that of patients with advanced CKD [12, 30].

\section{In vitro Studies: Cell Culture of Fibroblasts}

Serum from NSF patients has recently been shown to stimulate control fibroblast hyaluronan synthesis by up to 7 -fold and collagen by up to 2.4 -fold in comparison with control fibroblast cultures incubated with serum derived from healthy volunteers and dialysis patients not suffering from NSF. Control fibroblasts exposed to GdDTPA-BMA (1.0 mM) for up to 7 days showed a significant stimulation of proliferation but no response to incubation with $\mathrm{GdCl}_{3}$. The authors suggested that $\mathrm{Gd}^{3+}$ may not be responsible for the cell growth. Omniscan (GdDTPA-BMA) has also induced the expression of $\alpha$-smooth muscle actin staining, suggesting induction of a myofibroblast phenotype [31]. Additional studies comparing various $\mathrm{Gd}-\mathrm{CA}$ are required to elicit the existence of any difference in the effect on fibroblasts.

\section{How Can NSF Be Avoided?}

Patients at risk of NSF should be identified before administering Gd-CA. Screening for renal dysfunction by obtaining a history and/or laboratory tests should be implemented and is mandatory before the use of Omniscan, Optimark (non-ionic linear chelates) and Magnevist (ionic linear chelate). Imaging techniques that do not re- quire contrast medium injection should be considered in patients with marked reduction in renal function. If contrast-enhanced MRI examination with Gd-CA is deemed necessary in these patients, the following recommendations should be implemented [32].

- Patients with a glomerular filtration rate $\leq 30 \mathrm{ml} / \mathrm{min}$, including those on dialysis, should not receive nonionic linear chelates (Omniscan, Optimark) or the ionic linear chelate Gd-DTPA (Magnevist).

- The most stable Gd-CA should be used in these patients (macrocyclic chelates).

- The lowest possible dose of Gd-CA to achieve a diagnostic examination should be used.

- Allow at least 1 week before giving more Gd-CA.

- Avoid examination whenever possible during acute inflammatory events.

- Patients on haemodialysis can be scheduled to have the dialysis session shortly after the MRI examination.

- Patients on peritoneal dialysis are at extra risk due to slow elimination of Gd-CA. Several rapid exchanges should be encouraged to enhance the removal of the contrast molecules from the body.

\section{What Are the Current Recommendations of the Regulatory Authorities on the Use of Gd-CA in Patients with Renal Impairment?}

In the USA, the Food and Drug Administration has asked manufacturers of all Gd-CA to include a new boxed warning on the product label that states: 'Patients with severe kidney insufficiency who receive gadoliniumbased agents are at risk for developing a debilitating, and a potentially fatal disease known as nephrogenic systemic fibrosis (NSF).' In addition, it states that 'patients just before or just after liver transplantation, or those with chronic liver disease, are also at risk for developing NSF if they are experiencing kidney insufficiency of any severity'.

The Food and Drug Administration also recommends that patients should be screened for kidney problems prior to receiving any Gd-CA, the recommended dose should not be exceeded and enough time should elapse before the agent is used again.

In Europe, the UK Commission on Human Medicines and the European Pharmacovigilance Working Party of the Committee for Medicinal Products for Human Use recommend that: 
'Use of Omniscan (gadodiamide) is contraindicated in patients with severe renal impairment [i.e. GFR (glomerular filtration rate) or eGFR (estimated GFR) $<30 \mathrm{ml} / \mathrm{min} / 1.73 \mathrm{~m}^{2}$ ] or in patients with renal dysfunction who have had, or who are awaiting, liver transplantation. For patients with moderate renal impairment (i.e. GFR or eGFR $30-59 \mathrm{ml} / \mathrm{min} / 1.73 \mathrm{~m}^{2}$ ) or neonates and infants up to 1 year of age, Omniscan should be used only after careful consideration. Use of Magnevist (gadopentetic acid) is contraindicated in patients with severe renal impairment (i.e. GFR or eGFR $<30 \mathrm{ml} / \mathrm{min} / 1.73 \mathrm{~m}^{2}$ ). Magnevist should be used with caution in patients with moderate renal impairment (i.e. GFR or eGFR $30-59 \mathrm{ml} / \mathrm{min} / 1.73 \mathrm{~m}^{2}$ ), and should be used in neonates and infants up to 1 year of age only after careful consideration. All patients, particularly those older than 65 years, should be screened for renal dysfunction by obtaining a history and/or laboratory tests before these contrast agents are used. Careful consideration should be given to the use of the other gadoliniumcontaining MRI contrast agents in patients with severe renal impairment (i.e. GFR or eGFR $<30 \mathrm{ml} / \mathrm{min} / 1.73 \mathrm{~m}^{2}$ ).'

Further information can be found at the websites of the Food and Drug Administration (www.fda.gov/cder/ drug/infopage/gcca/default.htm), the UK Medicines and Healthcare Products Regulatory Agency (www.mhra.gov. uk), the European Society of Urogenital Radiology (www. esur.org) and the International Center for Nephrogenic Fibrosing Dermopathy Research (www.icnfdr.org).

\section{Is There an Effective Treatment for NSF?}

Several treatments (see below) have been evaluated, but none has shown consistent benefit. Therapy is usually directed towards improving renal function, hindering the progression of fibrosis and intensive physical therapy to prevent or reverse disability related to joint contractures.

\section{Renal Transplantation}

Stabilization or improvement of NSF is frequent in patients who recover renal function. Therefore, renal transplantation may benefit patients with NSF who are candidates for this procedure, although efficacy is not conclusively proven [33].

\section{Extracorporeal Photopheresis}

Extracorporeal photopheresis induces monocyte-derived tumour necrosis factor- $\alpha$, which in turn suppresses collagen synthesis and enhances collagenase production $[34,35]$. Although improvement of NSF symptoms was noted in some reports, the benefit of extracorporeal photopheresis in treating NSF remains unproven.

\section{Ultraviolet A1 Phototherapy}

Ultraviolet A1 phototherapy can inhibit procollagen synthesis in human skin, but its therapeutic effect has been inconsistent in treating NSF patients [36].

\section{Plasmapheresis}

The benefits of plasmapheresis in patients with NSF have been inconsistent and its therapeutic value is not certain [25].

\section{Pharmacological Manipulation}

Topical, intralesional or oral glucocorticoid therapy and cyclophosphamide have shown no benefit. Intravenous injection of sodium thiosulphate, which is an antioxidant and may have a chelating effect on free gadolinium, has been recommended by some authors, but the therapeutic efficacy of this drug remains uncertain [37].

\section{Conclusions}

NSF is a disease that may affect patients who have a marked reduction in renal function, including those on dialysis, and it has a strong association with exposure to low-stability Gd-CA. The reported prevalence of NSF after exposure to low-stability Gd-CA was around 5\% [6]. However, the true prevalence remains unclear. Recent reports documented high rates of incidence of NSF: 18\% according to Rydahl et al. [38], while Todd et al. [39] reported $30 \%$. The high prevalence of NSF in these studies could be due to identifying patients with mild disease which were probably overlooked in earlier reports. Systematic reviews of all patients with CKD exposed to GdCA are required to clarify the true prevalence of NSF.

There are further questions about NSF that remain unanswered and challenging for researchers in this field. The pathogenesis of NSF has not been established to date. The role of co-factors and whether they are independent risk factors that can initiate the process of NSF without the need for Gd-CA exposure is also not clear. Although there are few cases in the literature of NSF without a history of Gd-CA administration, exposure to Gd-CA cannot be excluded with certainty in these patients, particularly as their skin biopsies have not been examined for presence of gadolinium with sensitive techniques that detect even very small amounts of gadolinium [40].

It is hoped that the current efforts by researchers worldwide will improve our understanding of NSF and result in eradication of this devastating disease in the near future. 


\section{References}

-1 Cowper SE, Robin HS, Steinberg SM, Su LD, Gupta S, Leboit PE: Scleromyxoedema-like cutaneous diseases in renal-dialysis patients. Lancet 2000;356:1000-1001.

-2 Daram SR, Cortese CM, Bastani B: Nephrogenic fibrosing dermopathy/nephrogenic systemic fibrosis: report of a new case with literature review. Am J Kidney Dis 2005;46: 754-759.

-3 Cowper SE: Nephrogenic systemic fibrosis: a review and exploration of the role of gadolinium. Adv Dermatol 2007;23:131-154.

4 Cowper SE, Rabach M, Girardi M: Clinical and histological findings in nephrogenic systemic fibrosis. Eur J Radiol 2008;66:191-199.

$\checkmark 5$ Grobner T: Gadolinium - a specific trigger for the development of nephrogenic fibrosing dermopathy and nephrogenic systemic fibrosis? Nephrol Dial Transplant 2006;21: 1104-1108.

6 Marckmann P, Skov L, Rossen K, et al: Nephrogenic systemic fibrosis: suspected causative role of gadodiamide used for contrastenhanced magnetic resonance imaging. J Am Soc Nephrol 2006; 17:2359-2362.

7 Broome DR: Nephrogenic systemic fibrosis associated with gadolinium based contrast agents: a summary of the medical literature reporting. Eur J Radiol 2008;66:230-234.

-8 Marckmann P, Skov L, Rossen K, Heaf JG, Thomsen HS: Case-control study of gadodiamide-related nephrogenic systemic fibrosis. Nephrol Dial Transplant 2007;22:31743178.

9 Marckmann P, Skov L, Rossen K, Thomsen HS: Clinical manifestations of gadodiamiderelated nephrogenic systemic fibrosis. Clin Nephrol 2008;9:161-168.

10 Thomsen HS, Morcos SK, Dawson P: Is there a causal relation between the administration of gadolinium based contrast media and the development of nephrogenic systemic fibrosis (NSF)? Clin Radiol 2006;61:905-906.

-11 Penfield JG, Reilly RF: Nephrogenic systemic fibrosis risk: is there a difference between gadolinium-based contrast agents? Semin Dial 2008;21:129-134.

-12 Morcos SK: Nephrogenic systemic fibrosis following the administration of extracellular gadolinium based contrast agents: is the stability of the contrast agent molecule an important factor in the pathogenesis of this condition? Br J Radiol 2007;80:73-76.

-13 Morcos SK: Extracellular gadolinium contrast agents: differences in stability. Eur J Radiol 2008;66:175-179.

$\checkmark 14$ Idée JM, Port M, Raynal I, Schaefer M, Le Greneur S, Corot C: Clinical and biological consequences of transmetallation induced by contrast agents for magnetic resonance imaging: a review. Fundam Clin Pharmacol 2006;20:563-576.

15 Port M, Idée JM, Medina C, Robic C, Sabatou M, Corot C: Efficiency, thermodynamic and kinetic stability of marketed gadolinium chelates and their possible clinical consequences: a critical review. Biometals 2008, E-pub ahead of print.

16 Kimura J, Ishiguchi T, Matsuda J, Ohno R, Nakamura A, Kamei S, Ohno K, Kawamura T, Murata K: Human comparative study of zinc and copper excretion via urine after administration of magnetic resonance imaging contrast agents. Radiat Med 2005;23:322326.

17 Laurent S, Elst LV, Copoix F, Muller RN: Comparative study of the physicochemical properties of six clinical low molecular weight gadolinium contrast agents. Contrast Media Mol Imaging 2006;1:128-137.

18 Morcos SK, Thomsen HS, Webb JA; Contrast Media Safety Committee of the European Society of Urogenital Radiology (ESUR): Dialysis and contrast media. Eur Radiol 2002; 12:3026-3030

19 Perazella MA: Nephrogenic systemic fibrosis, kidney disease, and gadolinium: is there a link? Clin J Am Soc Nephrol 2007;2:200202.

20 Saab G, Abu-Alfa A: Nephrogenic systemic fibrosis - implications for nephrologists. Eur J Radiol 2008;66:208-212.

21 Sadowski EA, Bennett LK, Chan MR, Wentland AL, Garrett AL, Garrett RW, Djamali A: Nephrogenic systemic fibrosis: risk factors and incidence estimation. Radiology 2007;243:148-157.

22 Swaminathan S, Ahmed I, McCarthy JT, et al: Nephrogenic fibrosing dermopathy and high-dose erythropoietin therapy. Ann Intern Med 2006;145:234-235.

23 Boyd AS, Zic JA, Abraham JL: Gadolinium deposition in nephrogenic fibrosing dermopathy. J Am Acad Dermatol 2007;56:2730 .

24 Maloo M, Abt P, Kashyap R, et al: Nephrogenic systemic fibrosis among liver transplant recipients: a single institution experience and topic update. Am J Transplant 2006;6:2212-2217.

25 Baron PW, Cantos K, Hillebrand DJ, et al: Nephrogenic fibrosing dermopathy after liver transplantation successfully treated with plasmapheresis. Am J Dermatopathol 2003; 25:204.

26 Fazeli A, Lio PA, Liu V: Nephrogenic fibrosing dermopathy: are ACE inhibitors the missing link? Arch Dermatol 2004;140: 1401.

27 Sieber M, Pietsch H, Walter J, Haider W, Frenzel T, Weinmann HJ: A preclinical study to investigate the development of nephrogenic systemic fibrosis: a possible role for gadolinium-based contrast media. Invest Radiol 2008;43:65-75.

28 Sieber M, Lengsfeld P, Walter J, Schirmer H, Frenzel T, Siegmund F, Weinmann HJ, Pietsch H: Gadolinium-based contrast agents and their potential role in the pathogenesis of nephrogenic systemic fibrosis: the role of excess ligand. J Magn Reson Imaging 2008;27:955-962

29 White GW, Gibby WA, Tweedle MF: Comparison of Gd (DTPA-BMA) (Omniscan) versus Gd(HP-DO3A) (ProHance) relative to gadolinium retention in human bone tissue by inductively coupled plasma mass spectroscopy. Invest Radiol 2006;41:272-278.

30 Morcos SK: Validity of the animal models for nephrogenic systemic fibrosis. Invest Radiol 2008;43:338

31 Edward M, Quinn JA, Mukherjee S, Jensen MBV, Jardine AG, Mark PB, Burden AD: Gadodiamide contrast agent 'activates' fibroblasts: a possible cause of nephrogenic systemic fibrosis. J Pathol 2008;214:584-593.

32 Thomsen HS; European Society of Urogenital Radiology (ESUR): ESUR guideline: gadolinium-based contrast media and nephrogenic systemic fibrosis. Eur Radiol 2007;17: 2692-2696.

33 Cowper SE: Nephrogenic systemic fibrosis: the nosological and conceptual evolution of nephrogenic fibrosing dermopathy. Am J Kidney Dis 2005;46:763-765.

34 Lauchli S, Zortea-Caflisch C, Nestle FO, Burg G, Kempf W: Nephrogenic fibrosing dermopathy treated with extracorporeal photopheresis. Dermatology 2004;208:278280.

35 Gilliet M, Cozzio A, Burg G, Nestle FO: Successful treatment of three cases of nephrogenic fibrosing dermopathy with extracorporeal photopheresis. Br J Dermatol 2005; 152:531-536.

36 Fisher GJ, Datta S, Wang Z, et al: c-Jun-dependent inhibition of cutaneous procollagen transcription following ultraviolet irradiation is reversed by all-trans retinoic acid. J Clin Invest 2000;106:663.

37 Yerram P, Saab G, Karuparthi PR, Hayden MR, Khanna R: Nephrogenic systemic fibrosis: a mysterious disease in patients with renal failure - role of gadolinium-based contrast media in causation and the beneficial effect of intravenous sodium thiosulfate. Clin J Am Soc Nephrol 2007;2:258-263.

38 Rydahl C, Thomsen HS, Marckmann P: High prevalence of nephrogenic systemic fibrosis in chronic renal failure patients exposed to gadodiamide, a gadolinium-containing magnetic resonance contrast agent. Invest Radiol 2008;43:141-144.

39 Todd DJ, Kagan A, Chibnik LB, Kay J: Cutaneous changes of nephrogenic systemic fibrosis: predictor of early mortality and association with gadolinium exposure. Arthritis Rheum 2007;56:3433-3441.

-40 Abraham JL, Thakral C: Tissue distribution and kinetics of gadolinium and nephrogenic systemic fibrosis. Eur J Radiol 2008;66:200 207. 


\section{Editorial Comment}

M. El Nahas, Sheffield

This minireview by Morcos and Thomsen sheds new light on the emerging entity of nephrogenic systemic fibrosis induced by gadolinium-containing contrast media. It offers the readers answers to a number of outstanding issues and questions, including those related to the pathogenesis of this fascinating, and at times quite dramatic, condition that affects patients with advanced renal insufficiency injected with gadolinium-containing compounds used for MRI. The review also gives a synopsis of the latest recommendations and guidelines regarding the use of such material in CKD. Overall, this comprehensive and accessible review by 2 authorities on the subject is timely and informative 